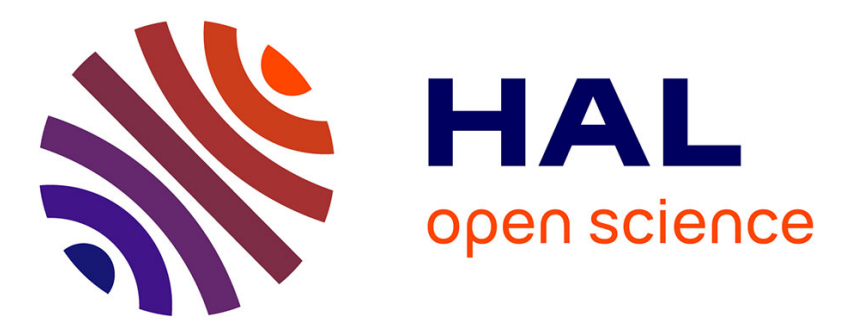

\title{
A microcontroller-based approach to monitoring pneumatic actuators
}

\author{
Mohammad Al Yami, Roger I Grosvenor, Paul Prickett
}

\section{To cite this version:}

Mohammad Al Yami, Roger I Grosvenor, Paul Prickett. A microcontroller-based approach to monitoring pneumatic actuators. International Journal of Production Research, 2010, 48 (11), pp.3193-3205. 10.1080/00207540902812102 . hal-00580107

\section{HAL Id: hal-00580107 https://hal.science/hal-00580107}

Submitted on 26 Mar 2011

HAL is a multi-disciplinary open access archive for the deposit and dissemination of scientific research documents, whether they are published or not. The documents may come from teaching and research institutions in France or abroad, or from public or private research centers.
L'archive ouverte pluridisciplinaire HAL, est destinée au dépôt et à la diffusion de documents scientifiques de niveau recherche, publiés ou non, émanant des établissements d'enseignement et de recherche français ou étrangers, des laboratoires publics ou privés. 


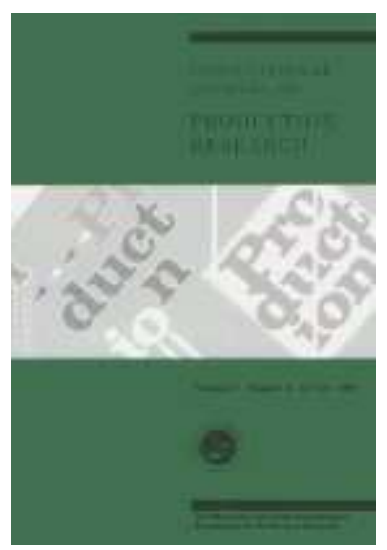

\section{A microcontroller-based approach to monitoring pneumatic actuators}

\begin{tabular}{|c|c|}
\hline Journal: & International Journal of Production Research \\
\hline Manuscript ID: & TPRS-2008-IJPR-0829.R1 \\
\hline Manuscript Type: & Original Manuscript \\
\hline $\begin{array}{r}\text { Date Submitted by the } \\
\text { Author: }\end{array}$ & 27-Jan-2009 \\
\hline Complete List of Authors: & $\begin{array}{l}\text { Al Yami, Mohammad; Cardiff School of Engineering } \\
\text { Grosvenor, Roger; Cardiff University, School of Engineering } \\
\text { Prickett, Paul; Cardiff School of Engineering }\end{array}$ \\
\hline Keywords: & $\begin{array}{l}\text { ASSEMBLY SYSTEMS, CONDITION MONITORING, FLEXIBLE } \\
\text { ASSEMBLY, QUALITY SYSTEMS }\end{array}$ \\
\hline Keywords (user): & ASSEMBLY SYSTEMS, CONDITION MONITORING \\
\hline
\end{tabular}

\section{scholarONE" \\ Manuscript Central}


1

2

3

4

5

6

7

8

9

\title{
A microcontroller-based approach to monitoring pneumatic actuators.
}

\author{
M. Alyami, R. I. Grosvenor and P. W. Prickett \\ Cardiff School of Engineering, Cardiff University \\ Queen's Buildings, Newport Road, \\ Cardiff CF24 3AA, Great Britain
}

\begin{abstract}
$\underline{\text { Abstract: }}$
This paper presents the development of a microcontroller-based process monitoring system that is capable of being embedded within high speed automated assembly systems. A generic architecture is presented to fully utilise the communication, data acquisition and signal processing attributes of a current generation microcontroller. The efficacy of this approach is illustrated by the development of an autonomous method in which the system acquires and analyses transient data related to the operation of a linear actuator. The performance of this system clearly identifies the potential of microcontroller based solutions as very powerful yet affordable real-time quality management tools. The low costs associated with the implementation offer the prospect of affordable integrated quality management to a wide range of industries.
\end{abstract}

\section{Keywords:}

Pneumatic actuator monitoring, pressure transient signal analysis, embedded microcontroller architecture, Digital Signal Controller (dsPIC). 


\section{Introduction}

Recent advances in embedded microcontroller technology make it possible to consider that low cost solutions may be developed to monitor the operation of devices based upon the deployment of pneumatic actuators. This research has generated solutions that can support much needed quality management functions for applications, such as part transfer systems, where a number of pneumatic devices are operating continuously in confined spaces and hazardous conditions. This paper describes the current findings of this research activity and describes the approach taken to engineer such a system.

Pneumatic linear actuators or cylinders are used in many manufacturing operations, including the automotive, manufacturing and food packaging industries. The main purpose of deploying such systems is to perform position control and enable parts handling, especially in robotic manipulators, end effectors and grippers. Such devices and systems often exhibit highly nonlinear characteristics mainly due to the compressibility of air and the variability of motion associated with the "stiction" which arises in cylinders. This makes achieving accurate dynamic position and stroke control difficult. These devices therefore tend to be limited to pre-set end position tasks. In these circumstances problems may arise due to part misalignment, incorrect workpiece selection, variability in air supply pressure and the obstruction of motion due to foreign objects.

The parameters that may be of interest when approaching the monitoring of such systems include the number and type of sensors needed, the nature of the computational resources required and the operating variables to be considered. Some work has been conducted into aspects of monitoring and controlling these devices, including research considering how the compressibility of air introduces a dead time (or delay) in the system's response that makes accurate control more challenging [Van Varseveld and Bone 1997 and Wang et.al 2004]. Air losses in cylinders and 
transmission lines have been shown to add more barriers to successful control [Messina et.al 2005]. These factors can make it difficult to achieve accurate position control without the use of added sensors. These sensors, and the controller needed to manage their operation, can be expensive.

The monitoring system outlined in this paper was developed based upon the data acquisition and processing capabilities of the current generation of microcontrollers. It was intended that the resulting system would be low cost, flexible and provide monitoring information for a range of applications and configurations. Advancements in microcontroller technology mean that current generation of devices can provide impressive levels of computational resources, as defined by processing power and computational speed, on a single device. The cost of such devices and their supporting circuitry typically vary between $£ 50$ and $£ 300$, depending upon the application being considered. This means that the development of a low cost distributed process monitoring system that offers real time diagnostic functionality can be considered. Previous experience indicates that investment in such a monitoring system will only be successful if it is reliable and can provide an acceptable cost-to-benefit ratio. In the case of the research described in this paper the attributes of the developed system, which include a plug-and-play capability, mean that it can provide a possible solution for a wide variety of monitoring applications.

Previous attempts have been reported based upon taking advantage of advances in low cost computing resources to exploit the recent innovations in field bus systems, microprocessors and computing technologies [Pu et al. 2000]. In this work the concept of intelligent component-based servo pneumatic actuation systems was advanced by designing templates for smart sensors, smart valves and smart actuators. It was proposed that limited intelligence can be embedded in such devices in order to provide information rather than raw data. These devices can then be integrated to form a smart pneumatic servo system capable of communication with the external world.

An analytical fault detection and diagnosis strategy for pneumatic systems was introduced [Li and Kao 2005] with the intention of preventing the failure of devices that can cause plant or 
process shutdowns resulting in the loss of production time and profits. The developed approach was signal-based, using parameters such as pressure and flow and employed an analysis methodology based upon wavelet decomposition. A pattern recognition technique was developed to diagnose unknown leakages and experiments were successfully carried out to test and verify the effectiveness of the proposed approach.

Another example of a fault detection and diagnostic system was developed for applications such as the mechanical systems operating pneumatic train doors commonly found in rapid transit systems [Lehrasab et al. 2002]. These doors are often controlled by solenoid valves and faulty operation can be a significant source of delay in railway services. The research demonstrated how the health of the system can be determined using feature extraction methodologies. This included throw and activation time characteristics, spectral features of the door displacement profile as well as abstract features such as velocity profiles and pressure drop during the throw. Once a fault was indicated neural network models were utilised for diagnosis. The authors suggested the implementation of this approach in a distributed architecture and proposed that diagnostic results be integrated into the maintenance information system in order for a proactive maintenance regime to be produced.

Neural network enabled fault detection and identification was the basis of a system developed to manage actuator faults in a process control valve [Karpenko et al. 2003]. A software package supplied by the valve manufacturer was used to communicate with onboard sensors and obtain its' position response under various operating conditions. Fault signatures associated with incorrect supply pressure, actuator vent blockage and diaphragm leakage were considered. Experimental data was collected and neural networks trained, and tested. The authors claimed to be successful in the detection and identification of various magnitudes of the faults of interest.

The research outlined above supports the potential for developing low cost monitoring approaches that can be applied to pneumatically operated parts handling operations. However, most 
of the reported research solutions are not suited to such applications, either due to cost considerations or due to the time needed for diagnosis. This paper describes the development of a distributed process monitoring system which may be deployed to oversee operations based upon pneumatic actuators and illustrates its efficacy with an example application.

\section{Monitoring System Architecture}

The term "distributed system" is used here in to describe a system in which interconnected hardware or software components communicate and coordinate their actions. The main benefits of such systems include their robustness and reliability; since processing power is distributed the failure of a single element does not disable the entire system. Data can be maintained at different locations to further improve system reliability. Other attributes include functionality and scalability. Since the system may incorporate various types of devices each element may be selected to optimise the function it is expected to perform. Additional task specific elements may be added as required. These attributes mean that the devices deployed in a distributed system can operate concurrently to undertake monitoring actions independently. Such actions must however be coordinated within suitably structured system architecture.

The Intelligent Process Monitoring and Management (IPMM) research group at Cardiff School of Engineering has previously exploited the capabilities of the 8-bit microcontrollers in a range of monitoring applications; including the monitoring of machine tools [Amer et.al. 2006] and process plant [Ahsan et.al. 2006]. The advantages of such devices may be considered in terms of their low cost and ease of use. The limitations of such devices, most specifically measured in terms of their processing capabilities, may have previously restricted their application. However the current generation of microcontroller devices, such as those used in this research, has overcome such limitations. In particular the availability of the new more powerful microcontrollers and 
similar devices seems to offer an almost limitless potential in the engineering of product quality and process management and monitoring systems [Siddiqui et.al. 2007].

To support effective monitoring real-time information about the enactment of the process and the condition of selected machine components is needed. A fundamental aspect of the deployment of a monitoring system is data acquisition, which is normally enabled through the installation of various sensors and transducers. An industrial application may require a large number of such sensors. If this data is collected at a central location, communication infrastructure will have to be provided from every sensing element. This approach can lead to a complex communication network and result in a system that is hard to install and difficult to maintain.

Advances in wireless technologies have supported developments in this area, but the cost and data transmission limitations associated with such systems remains an important issue to consider. The handling of the data acquired from sensors in such applications tends to be very challenging. Data files can become very large and the communications infrastructure has to cope with the often continuous nature of much of the data. For example, the monitoring of a six station press line with a four stage part transfer system per station operating on a three second cycle, could result in some two million separate actuator motions per week. The effectiveness of the monitoring system may thus be compromised by the quantity of data to be acquired, processed and communicated. A modular, microcontroller-based, data acquisition and analysis system was therefore developed to provide a more applicable solution. The key aim in this system is the intelligent acquisition and analysis of data and the determination of any associated fault diagnosis at source, thus massively reducing data communication and storage requirements.

Another important objective of this research was to develop and deploy data processing techniques capable of monitoring the operation of a particular machine function or activity, whilst keeping the number of added electronic components and sensors to a minimum. The hardware architecture developed in previous IPMM research is shown in Figure 1. It was decided to deploy 
the 16-bit dsPIC microcontrollers in the further development of this system. Their built-in digital signal processor and computational speed make them superior to the previous generations of microcontrollers, thereby promising considerable additional benefits to fault diagnosis.

Figure 1. IPMM Microcontroller based monitoring system architecture.

\subsection{The Front End Node (FEN)}

The key feature required in this approach was processing speed. The device selected for both the front-end node of the distributed process monitoring system had five (16-bit) timers; two of which can be paired to form a 32-bit timer if and when required. An input capture module, wellsuited for applications where the period or frequency measurement is required, is also available on this chip. The microcontroller offers several I/O ports supporting multiple serial and parallel communication protocols. In this research communication between the nodes was supported by the adoption of a Controller Area Network (CAN) infrastructure which is employed in many areas of industrial automation.

The CPU of this device has a 16-bit (data) modified Harvard architecture. It provides 144Kbytes of program memory, $4 \mathrm{Kbytes}$ of data EEPROM memory. It's possible to execute all instructions in a single cycle. The architecture supports program loop constructs through the use of DO and REPEAT instructions, yielding no program overhead. The CPU supports various flexible addressing modes to meet compiler needs; for most instructions the devices have the capability to perform a data (or a program) memory read, a data memory write and a working register (data) read in a single instruction cycle. The essential feature of the acquisition process supporting this development is that analogue signals have to be converted into a more convenient format so that they can be analysed in a meaningful and efficient manner as near to their source as is practical. This is achieved by means of the on board 12-bit (16 channel) analogue-to-digital converter. 
In the developed approach any desired parameter can be connected to a suitably configured FEN. When processed the acquired digital data can be very quickly analysed using the same microcontroller to identify "pre-defined" known fault symptoms. If required the processed information can then be sent through the CAN bus to collaborating FENs. Signal acquisition and processing are thus performed in this layer of the monitoring system. It is important to state that data generated from each monitoring action will be analysed for possible faults and, if none are identified, the data is summarized and sent to a buffer. This retains data that can be interrogated should a fault condition occur, but, as more data is generated the previously analysed data which relates to normally enacted operations can be discarded.

\subsection{Data Acquisition}

The first step in the operation of the FENs is to acquire the required signals from different sensors and transducers. Various sensors are usually installed in the process or machine to perform such a task. In most cases the output measurement of a sensor requires signal conditioning to conform to the requirements of the data acquisition hardware. In the context of this research for example a typical pressure transducer output would be a 4-20 mA current signal. This signal had to be converted to voltage in the range 0 to $5 \mathrm{VDC}$ as required by the dsPIC. The resulting signal then needs to be converted into digital format for processing using the ADC module embedded in the dsPIC. This employs the concept of successive approximation, which is the fastest and most commonly used technique in analogue to digital conversion.

When using ADC some parameters have to be considered in order for a process monitoring and control system to be most effective and robust. Those of major importance include resolution; the available ADC converter has a 12-bit resolution, which provides 4096 levels of representation of the analogue signal. Another consideration is the sampling rate, which should be at least twice the maximum frequency content of the signal to avoid aliasing [Smith, 1999]. For practical reasons, analogue signals are actually sampled at an even higher rate to account for the differences in 
performance between real-world ADC and the ideal one [Huddleston, 2007]. In this application the pressure signal was sampled at $2 \mathrm{KHz}$, which is five times the highest frequency content as empirically determined. Similar levels of capability were possible in the linearity, repeatability and range of the input signals.

The second types of signals that are of interest to monitoring systems are digital signals. These can be acquired to provide information about discrete events in the process or machine such as the on/off state of a valve or a limit switch. Once these were interfaced with the microcontroller's timer modules it was possible to measure the time between discrete events.

\subsection{The Connectivity node}

The Connectivity node, which forms the second level in the distributed monitoring system architecture shown in Figure 1, has two functions. Firstly it is required to co-ordinate FEN activity in cases where the fault detection process proves to be challenging. Such co-ordination is critical under circumstances which require that information from various FENs be shared in order to reach a conclusive decision about the health of monitored parameters. For instance, if a node in a pneumatic system determines that the air pressure is too low, a message may be sent to notify other nodes in the system that may mistake the consequences of low pressure on the element they are monitoring as a fault. This can minimise the number of false alarms generated. In the deployed system possible faults and/or the associated data from various front-end-nodes can be combined and analysed. It's believed that eventually all remaining "known" fault conditions can be detected at this level.

The second function is to provide the communication link with the outside world. The adopted Ethernet based solution was implemented in a modular fashion following the TCP/IP protocol. It was thus designed to be independent of operating systems and to be capable of cooperative multitasking. This level is therefore used to provide a link between the FENs and a more powerful computer residing in level three of the system. Confirmed faults and associated 
operational information can be communicated to interested users and management functions embedded at level three for action and storage. In this way further analysis tools and expert-user knowledge can be deployed to identify the occurrence of "new" faults as they arise. Any new faults thus detected and diagnosed together with steps aimed at subsequent fault recognition may then be added to the list of known faults, and the developed detection process added to the capabilities of the FENs.

In practice the second level of the architecture shown in Figure 1 acts as a service level by providing and managing these communication tasks. Since the high performance microcontroller selected as the FEN module had the capacity to undertake these tasks it was determined that level two activities could be incorporated into the FENs. As such the FEN that first detects a possible fault condition assumes the role of the Connectivity node, and oversees all level two functions. All FENs can act in this way, providing a robustness and flexibility that takes full advantages of the capabilities of the deployed microcontroller devices. The resulting low-cost monitoring system has excellent capabilities in terms of signal acquisition and processing, a high level of integration, processing power, and communication facilities. Section three of this paper presents an example application of this system.

\subsection{Level Three functions}

Higher level management functions are undertaken at level three of the system. These functions may be undertaken locally or remotely. They will include the diagnosis of faults that require more advanced tools, and the continuing development of monitoring capabilities based upon the accrued data and information gathered from the deployed microcontrollers. It is important to note that any remote user, with the correct level of authority, may access any of these nodes directly. This supports the ability to monitor device operation in real time and provides access to all buffered data to support fault diagnosis. This facilitates the continuous improvement of the diagnostic ability of each node and of the overall system. 


\section{Experimental test set-up}

The test rig designed and constructed to research the development of this monitoring method is shown in Figure 2. The Air Preparation unit (A) conditions the air by trapping moisture, removing impurities and adding lubrication. It also contained a regulator to maintain the supply pressure at a constant level. The Solenoid Valve (B) was chosen due to its high flow capacity, response speed, low power consumption and long life expectancy. The Pressure Transducer (C) measures the line pressure of the air supplied from the Air Receiver (D). The pressurised air is supplied to the Actuator (E), through the Solenoid Valve. The monitoring functions are integrated into the development board $(\mathrm{F})$

Figure 2. Linear Actuator Test Rig.

In the application under consideration in this research the conditioned pressure transducer signal was connected to the $\mathrm{ADC}$ converter and sampled at a rate of 256 samples-per-second. The ADC was configured for one sample per interrupt, and its clock was derived from the system's oscillator. Data was transmitted as soon as a value becomes available. One of the microcontroller's digital outputs was used for the switch controlling the solenoid valve. An electronic switch was designed to control the operation of the valve. A $15 \mathrm{~V}$ DC supply is used to power the solenoid valve. A simple control algorithm was implemented to perform this testing. LEDs on the expansion board were used to represent the status of the valve and to indicate faults to the operator whenever they occur. The output sample from the ADC was scaled for an on-board LCD screen. Using the built in UART2 communications module data was transmitted to a PC allowing Microsoft Excel to be used for initial data analysis to develop the monitoring system. The same link was subsequently used to evaluate the effectiveness of the monitoring system in determining the health of the process. 
In order to achieve the required speed of response it was determined to base the approach on transient events.

The linear actuator used was a high speed precision device with a stroke of up to $100 \mathrm{~mm}$ able to apply loads of up to $200 \mathrm{~N}$. Precise adjustment of the mechanical end-position of the slide motion was possible. To facilitate the initial calibration tests this actuator was initially fitted with two plug-on proximity sensing units to indicate the defined actuator end-position. The sensors used on this rig operate with a supply voltage ranging from 8 to $30 \mathrm{VDC}$, with a maximum load current equal to $200 \mathrm{~mA}$.

The operating pressures used for the testing ranged from 3 to 7 bar. The intention of this research was to produce a monitoring system that operates at any pressure within this range. This accommodates any possible set-up of the system and allows for localized changes in supply pressure in any potential implementation. The experimental procedure adopted in this work initially set the air supply pressure to a series of specific values. A set of test pieces of known dimensions were used to restrict movement to a known end position. The calibrated test piece was placed to limit the stroke and the end position was measured. At a given supply pressure, the time taken by the slide unit to reach an object was then measured. A relationship between the travel time and end of stroke was then realized. In this way, a time based monitoring system was developed. If the travel time at a specific supply pressure and intended end position does not match to a defined tolerance the pre-set value, assuming the same operating conditions, a faulty situation is indicated to the operator.

\subsection{Signal Acquisition and Analysis.}

To develop a robust and flexible monitoring solution, the system was evolved through three stages. Initially two limit-switches were used to monitor the home and end positions of the actuator. This provided details of stroke times and allowed the initialization of appropriate data acquisition and analysis tools. The next step removed one sensor and the pressure response at the inlet of the 
actuator was acquired and analysed. The patterns associated with the operation of the actuator were then used to provide potential performance indicators. Finally, the pressure transient signal alone was monitored.

The data leading to the development of the technique capable of identifying the slide end position or the object size was initially acquired using the microcontroller. The output signal from the pressure transducer was converted into the correct format and the pressure samples were recorded and stored in an array. Data was then transmitted to a PC for analysis. This approach monitored only the pressure transient at the inlet to the actuator's cylinder. In this way, the cost of the monitoring system is reduced. In addition to monitoring actual actuator travel it was anticipated that the pressure signal measurements would also identify any faults arising during an operation.

Figure 3 illustrates the pressurization/actuation cycle for a test conducted at 5 bars with a $20 \mathrm{~mm}$ test piece in position. It is typical of such tests and shows the effect of the response time of the solenoid valve, which causes a delay in actuation from the trigger point (Time " 0 " on the figure). Once the valve is triggered, it can be seen that air pressure takes some time to build up to provide sufficient force to overcome friction and push the slide piston from its home position. The transient overshoots before starting to settle during the slide motion; once it reaches the test piece resistance to motion is encountered leading to a decreasing oscillation in the pressure signal, which dies out with time. As soon as the valve receives the signal to close, air exits the cylinder to the atmosphere depending on the valve's response time and the pressure falls rapidly to zero.

\section{Figure 3 . Travel time computation at 5 bar using a $20 \mathrm{~mm}$ test piece.}

In order to simplify the monitoring task it was desirable that the sample peak of the pressure signal should be used to reference the effective start of the motion. It was observed from the calibration tests using the limit sensors that the sample peak always occurred a fixed time after the actual start of the motion. The actual elapsed time was found to vary in a linear fashion with test 
pressure. This behaviour effectively "fixed" the start point of the motion sensing procedure to the occurrence of the peak pressure signal.

The second point needed to establish travel time must clearly correspond to the instant at which motion ceases, defined in this work as the end position. For clarity in Figure 3 the high frequency component of the pressure signal has been smoothed using a moving average filter. A filtering process was also deployed on the raw signal to extract the dominant component information. Careful investigation of responses for different end of stroke positions at each pressure identified that the squared rate of change could be used to identify the motion end point. Figure 3 again illustrates the procedure developed. The squared rate of change plot exhibits a clear peak corresponding to contact with the test piece. By establishing a threshold the designated "contact point" can easily be determined. Once again, through the initial calibration of this motion using end position sensors, good correlation was found between the established threshold point and the actual motion end point. The threshold value is shown in Figure 3 at 400 for a test at 5 bar This threshold was used except for pressures above 4 bar; the threshold below four bar was 250 to 300 .

The microcontroller device deployed in this research was selected to provide the processing power and mathematical capability needed to identify both the start point and end point from the two samples and complete the analysis of travel times fast enough to facilitate effective process monitoring. This is a relatively simple procedure; the travel time is computed as the difference between contact time and home position time. In all tests the sampling rate used was $2 \mathrm{kHz}$. This procedure was applied for each supply pressure acting upon multiple objects.

Curve-fitting procedures were developed to obtain a surface capable of acting as a process model using a non-linear least squares technique to fit the actual data. Figure 4 shows the surface representing the resulting travel time measurements. The concept being developed in this research is to model this surface to allow predicted travel times to be generated for any combination of pressure and end position. 
1

2

3

4

5

6

7

8

9

10

11

12

13

14

\section{Figure 4. The generated surface relating travel time, end position \& pressure.}

The surface model function used was of the form;

$$
z=\frac{A+x}{B+C y}+D y
$$

Where, $x$ is the end position in millimetres, $y$ the applied pressure in bar, and $z$ the travel time in milliseconds. The modelling procedure was found to converge after eight iterations at which point the parameters were estimated. The model was then exported to a more powerful mathematical modelling process to tune the parameters so that the best possible fit is achieved with a single set of parameters per surface. In the set up presented here the fitting parameters obtained were:

$$
\mathrm{A}=-102.3, \mathrm{~B}=-0.53, \mathrm{C}=-0.43 \text { and } \mathrm{D}=0.9 \text {. }
$$

The actual surface and the model were found to correlate well. Their computed correlation coefficient is 0.998 , which signifies the goodness of the model performance. The model performs well in the estimation of travel time as compared to the time obtained from information extracted from the pressure transient.

\section{Monitoring System Development}

The system outlined above was applied to the monitoring of actuator operations running at rates of four cycles per second. The maximum motion tested was $\pm 100 \mathrm{~mm}$, and it was found that the actuator end position could be determined with a precision of $1 \mathrm{~mm}$. Hundreds of cycle tests were undertaken for each of eight different end positions at a number of known pressures. In each case the end position was correctly assessed by the system before the actuator returned to the "start" position. It was thus established that the system had met an important requirement; that diagnosis of an error should be possible before the commencement of the next cycle. These tests established that 
the system was able to operate flexibly with different end positions, which reflects the needs of "pick and place" production systems.

In order to deploy the system within a production environment it is proposed that an operator will initially supervise a number of test cycles to calibrate the system to introduce a new product or set up and if required supervise the adjustment to the estimation parameters to suit the application in hand. To test this facility, actuator operations were undertaken at a series of “intermediate" pressures, i.e. 4.75 and 5.75 bar, for which no previous tests had been performed. Since the performance surface for the actuator was embedded within the microcontroller it was possible for the system to match the anticipated performance with that measured to indicate satisfactory operations. This procedure was undertaken at level one of the deployed monitoring system. At this level the operator communicates directly with the dsPIC via simple controls. Operator involvement was necessary in these initial tests to effectively establish the calibration of the new set-up by confirming that a correct operation had occurred. It was found that between ten and fifteen such cycles were sufficient to position the new set-up on the existing surface, following which unsupervised operation could continue. It should be noted that, once such an activity has been enacted, the results are added to the existing surface information and the activity does not have to be repeated. This is vital in a production environment, where several process variations may be normally present. Using this system each variation will be captured upon its first implementation and saved for future access. Similar actions were applied to the introduction of "new" end positions.

In many industrial settings the same operator can be required to oversee several elements of the process. Using this system it is possible that operator attention can be immediately drawn to any faults arising in the processes in real time so that prompt action can be taken, thus reducing operational cost and optimising performance. When needed to, for example following the installation of a new or replacement actuator, the operator can also interact with the system to redefine surface parameters and to manage the integration of the entire monitoring function based 
upon the collective expertise of each element retained within the system memory. This local control is represented by level two in the architecture, through which access may be gained to previously created performance surfaces stored within the management level PC. This ensures that the monitoring of any re-configured pneumatic systems can benefit from previously acquired performance surface information relating to the actuator hardware deployed. This facility is also important if more advanced fault detection and process management tools are to be deployed. The architecture facilitates the adoption of a "plug and play" facility, allowing the system to expand as required and supporting a flexible approach to process monitoring. This allows the surface models embedded within the devices to be continuously improved to accommodate different process setups, allow for different actuators etc. Fault diagnosis at this level can also help the development of improved process design, operation and management.

The research reported in this paper supports the deployment of a pressure based fault diagnosis system as a potential solution to monitoring the performance of any parts handling systems that are based upon linear pneumatic actuators. Initial tests have shown that the approach is both flexible and robust and can support reliable fault diagnosis. Most importantly the processing speed and power of the deployed microcontrollers means that this diagnosis can be accurately made in real time. In the tests outlined above diagnosis was achieved for cycle times of 0.25 seconds. In practice the microcontroller at the heart of this system operates at the micro-second level and can respond much faster than this if needs be. This suggests that the approach can accommodate the needs of many such implementations, potentially including high speed production processes. It also indicates that a single node may actually be used to monitor a number of linked actuator actions, with the individual performance surfaces being accessed as appropriate. Further investigations into the implications of these findings are ongoing but the expectation is that a single module will be capable of monitoring the operation of a complete transfer system containing multiple actuators. 


\section{References}

Ahsan, Q., Grosvenor, R.I. and Prickett, P.W. 2006. A distributed on-line system for process plant monitoring. Proceedings of the Institution of Mechanical Engineers Part E, Journal of Process Mechanical Engineering, 220, 61-77.

Amer,W., Grosvenor, R.I. and Prickett, P.W. 2006. Sweeping Filter and Tooth Rotation Energy Estimation (TREE) Techniques for Machine Tool Condition Monitoring. International Journal of Machine Tools \& Manufacture, 46, 1045-1052.

Huddleston, C. 2007. Intelligent Sensor Design Using the Microchip dsPIC. ButterworthHeinemann Ltd, ISBN: 0-7506-7755-4. 46-47.

Karpenko,M., Sepehri, N. and Scuse, D. 2003. Diagnosis of process valve actuator faults using a multilayer neural network. Control Engineering Practice, 11 (Nov),1289-1299.

Lehrasab, N., Dassanayake, H.P.B., Roberts, C., Fararooy, S. and Goodman C.J. 2002. Industrial fault diagnosis: Pneumatic train door case study. Proceedings of the Institution of Mechanical Engineers, Part F: Journal of Rail and Rapid Transit, 216, 175-183.

Li, X. and Kao, I. 2005. Analytical fault detection and diagnosis (FDD) for pneumatic systems in robotics and manufacturing automation, IEEE/RSJ International Conference on Intelligent Robots and Systems, August 2-6, Edmonton, Canada, 2517-2522.

Messina,A, Giannoccaro,N.I. and Gentile, A. 2005. Experimenting and Modelling the Dynamics of Pneumatic Actuators Controlled by the Pulse Width Modulation (PWM) Technique. Mechatronics, $15,859-881$.

Pu, J., Moore, P.R. and Wong, C.B. 2000. Smart components-based servo pneumatic actuation systems," Microprocessors and Microsystems, 24, 113-119.

Smith, S.W. 1999. The Scientist and Engineer's Guide to Digital Signal Processing. California Technical Publishing, ISBN: 0-9660176-6-8., 41-42. 
Siddiqui R.A., Amer W., Ahsan Q., Grosvenor R.I. and Prickett P.W. 2007. Multi-band infinite impulse response filtering using microcontrollers for e-Monitoring applications. Microprocessors and Microsystems, 31, 370-380.

Van Varseveld,R.B and Bone, G.M. 1997. Accurate Position Control of a Pneumatic Actuator Using on/off Solenoid Valves. IEEE/ASME Transactions on Mechatronics, 2(3),195-204.

Wang, J., Wang, J. D, Daw, N. and Wu, Q.H. 2004. Identification of Pneumatic Cylinder Friction Parameters using Genetic Algorithm. IEEE/ASME Transaction on Mechatronics, 9(1),100-107.

\section{ACKNOWLEDGEMENT:}

M. Alyami is a PhD student sponsored by the Royal Commission of Jubail, Saudi Arabia.

Formatted: Left, Line spacing: Double 


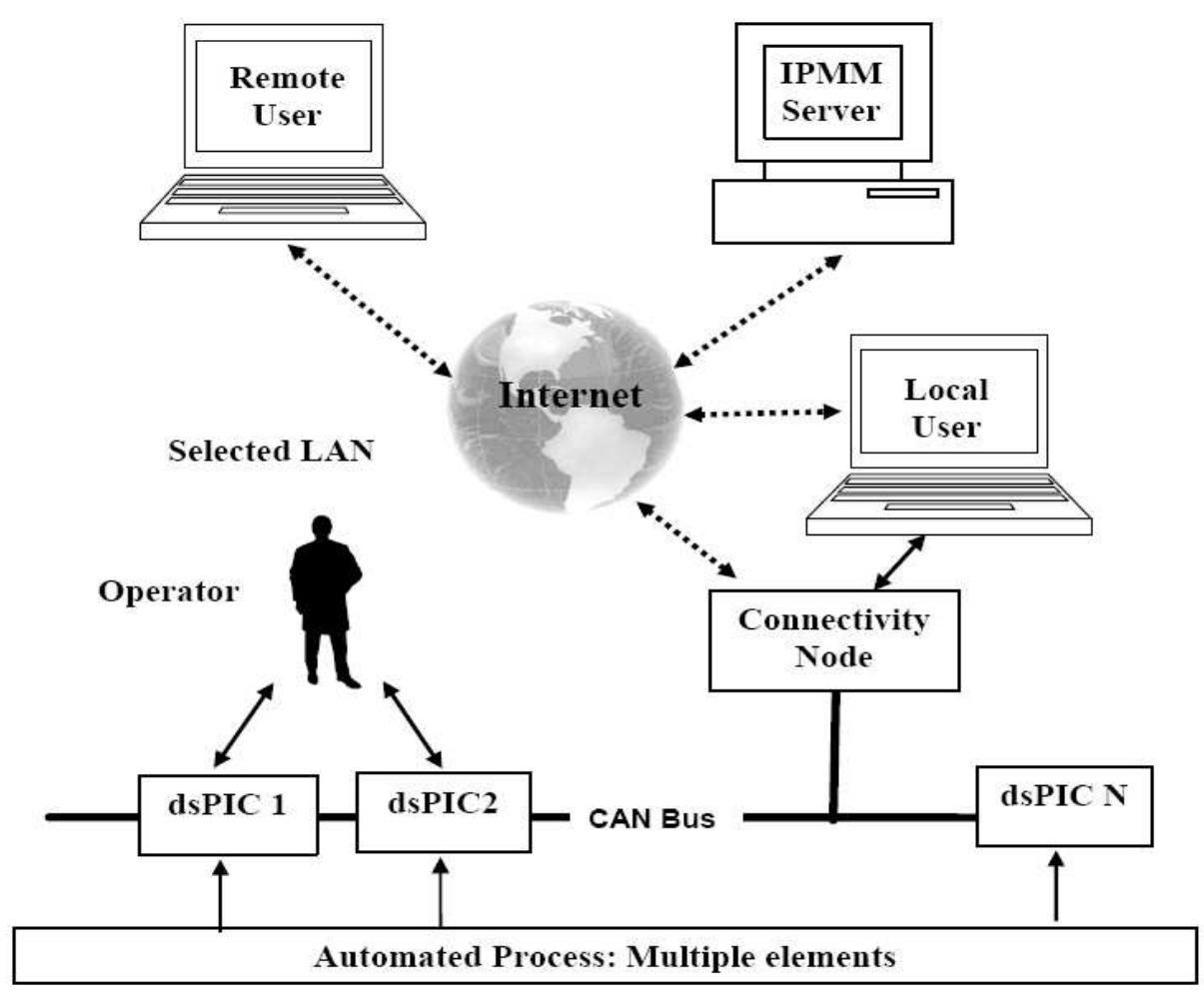

Figure 1. IPMM Microcontroller based monitoring system architecture. 


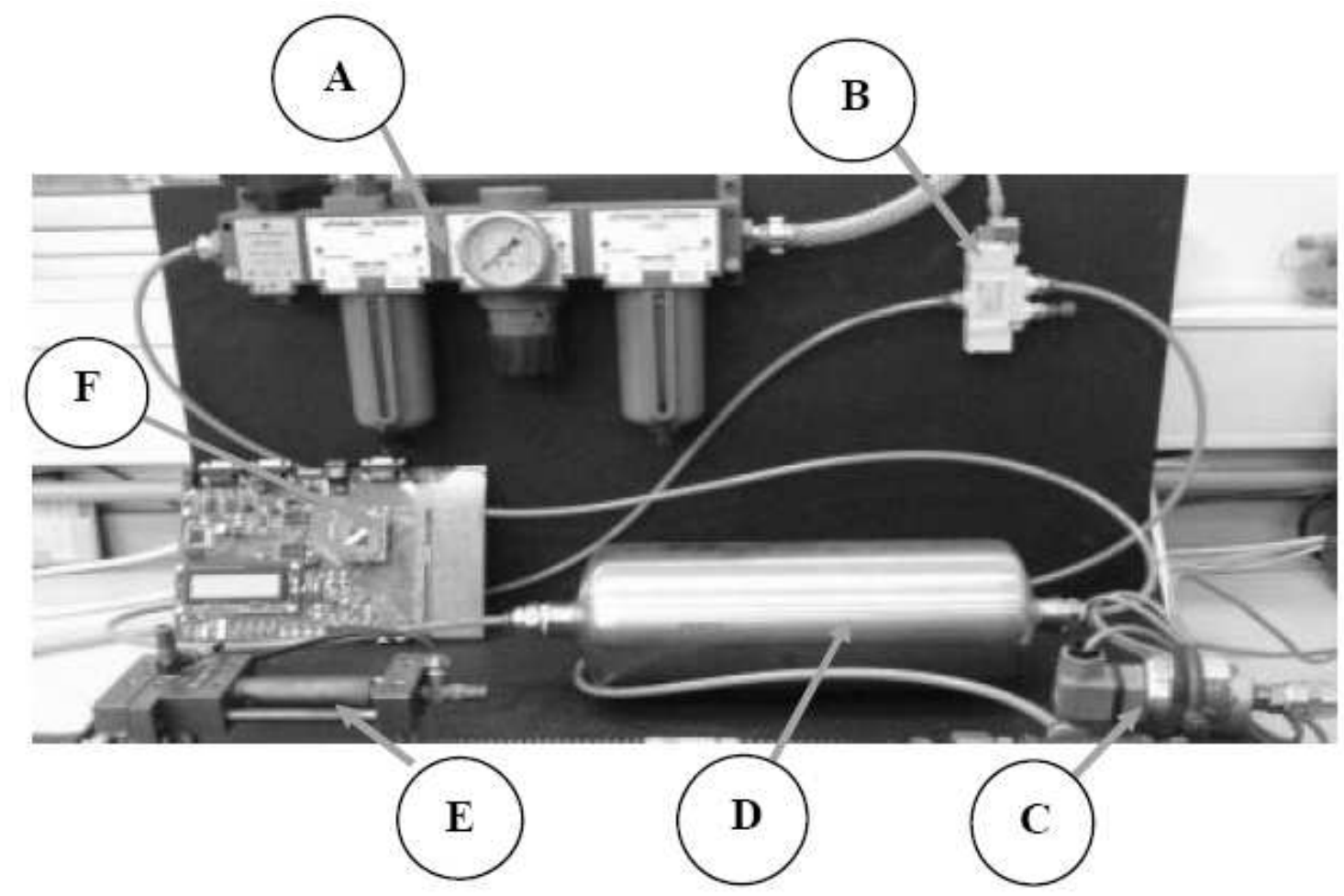

Figure 2. Linear Actuator Test Rig. 


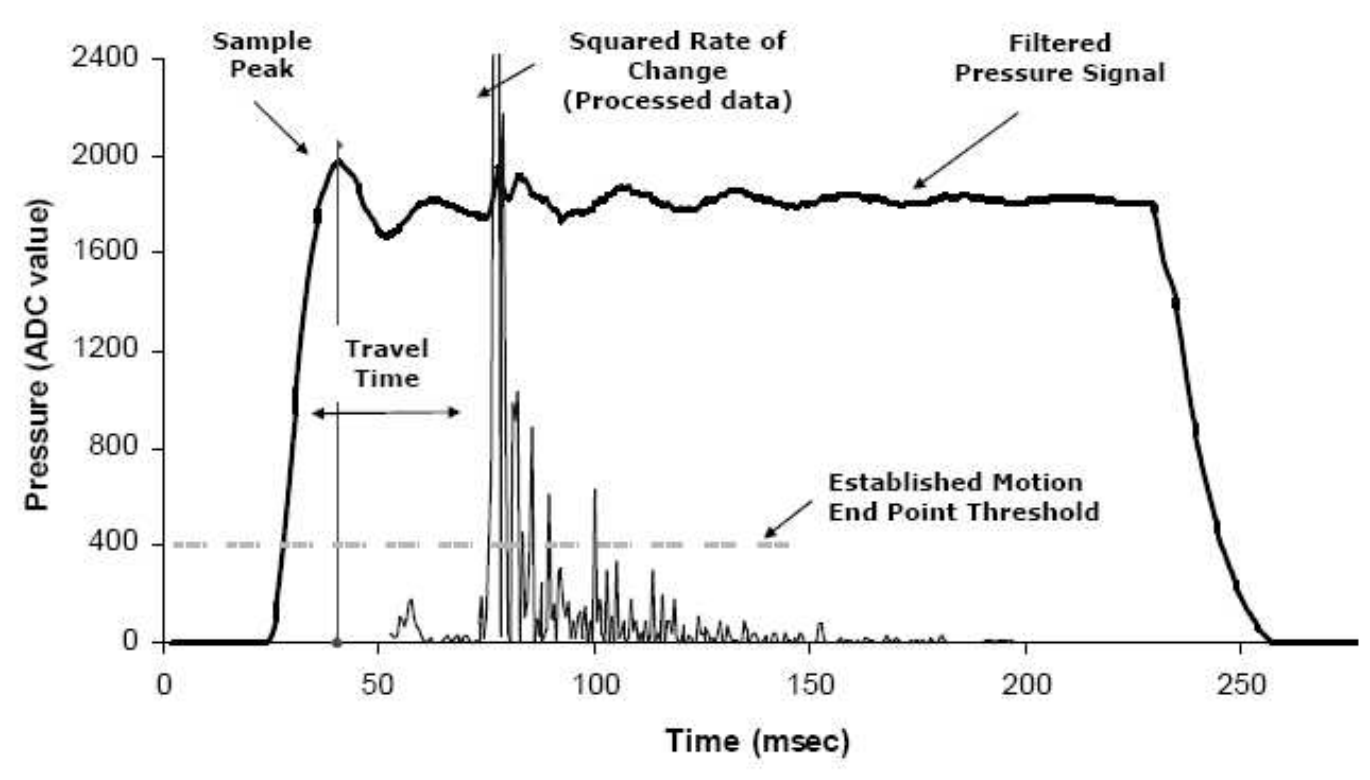

Figure 3. Travel time computation at 5 bar using a $20 \mathrm{~mm}$ test piece.

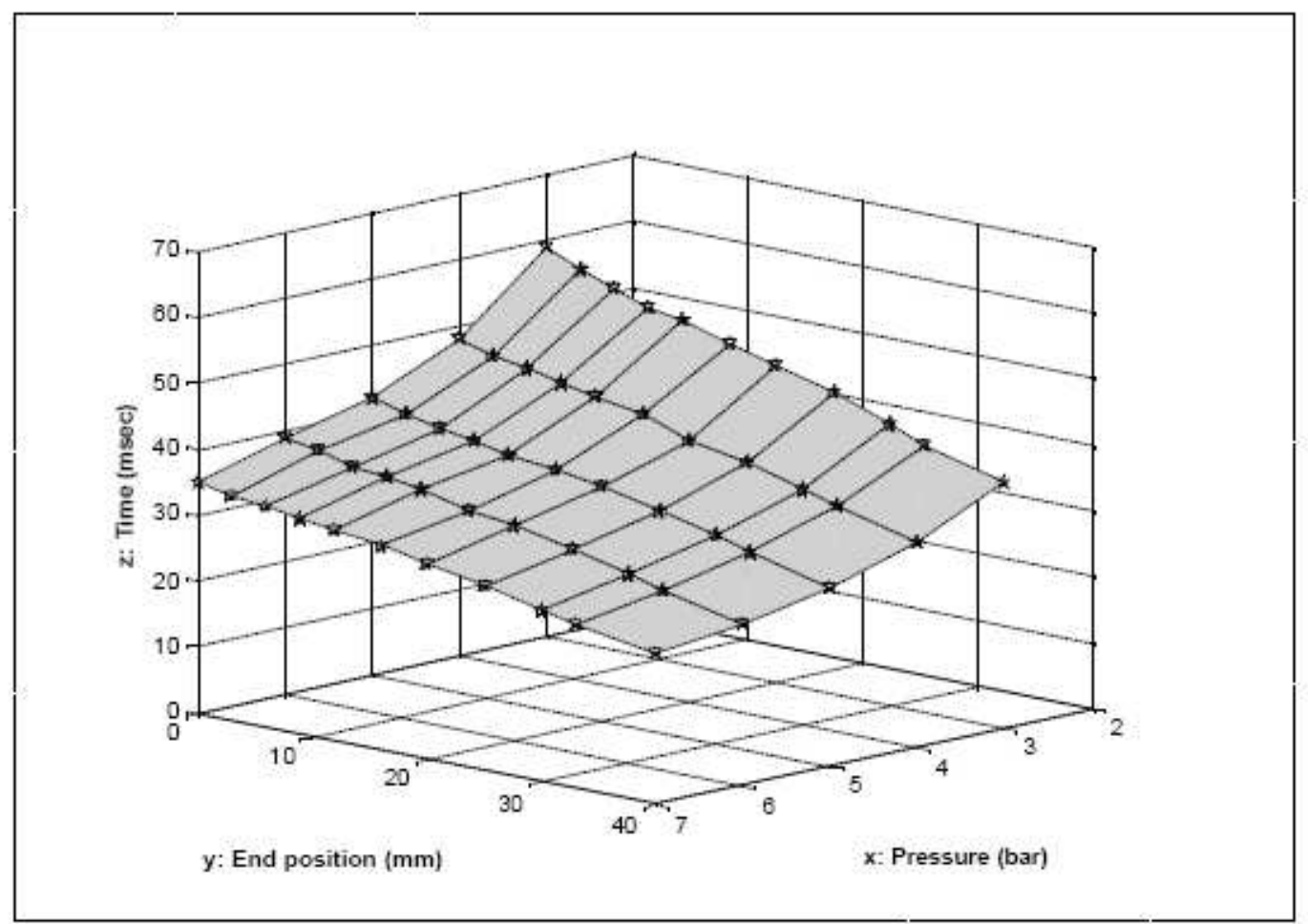

Figure 4 . The generated surface relating travel time, end position \& pressure. 